\title{
Efficacy of artemisinin combination therapy for the treatment of uncomplicated falciparum malaria in Nigerian children
}

\author{
Olusola Ojurongbe ${ }^{1,2}$, Olubunmi A Lawal ${ }^{3}$, Oyindamola O Abiodun ${ }^{4}$, John A Okeniyi ${ }^{3}$, Ayobami J \\ Oyeniyi $^{5}$, Oyeku A Oyelami ${ }^{3}$ \\ ${ }^{1}$ Department of Medical Microbiology and Parasitology, Ladoke Akintola University of Technology, Osogbo, Nigeria \\ ${ }^{2}$ Malaria Research Clinic and Laboratory, Ladoke Akintola University of Technology, Osogbo, Nigeria \\ ${ }^{3}$ Department of Paediatrics, Wesley Guild Hospital, Ilesa, Nigeria \\ ${ }^{4}$ Department of Pharmacology and Therapeutics, College of Medicine, University of Ibadan, Ibadan, Nigeria \\ ${ }^{5}$ Department of Family Medicine, Wesley Guild Hospital, Ilesa, Nigeria
}

\begin{abstract}
Introduction: The development and spread of Plasmodium falciparum resistance to most commonly used antimalarials remain a major challenge in the control of malaria. Constant monitoring of drug efficacy is an important tool in establishing rational antimalarial drug policies.

Methodology: A randomized comparative study was conducted at the Wesley Guild Hospital, Ilesa, Nigeria between February 2010 and September 2011 comparing the efficacy and safety of artemether-lumefantrine (Coartem) and fixed dose of artesunate plus amodiaquine (Larimal) in the treatment of uncomplicated P. falciparum malaria in children betweem 6 and 144 months of age. P. falciparum malaria parasitemia was assessed by microscopy and rapid diagnostic test. Drugs were administered according to age for three days under supervision. The primary efficacy endpoint was a day 28 PCR-corrected parasitological cure.

Results: A total of 182 patients were enrolled in the two treatment groups, Coartem $(\mathrm{n}=101)$ and Larimal $(\mathrm{n}=81)$, and tested after 28 days. In the intention-to-treat population, Coartem $(n=101)$ and Larimal $(n=81)$ had a PCR-corrected cure rate of $98 \%$ and $100 \%$ respectively, while in the per-protocol population, Coartem $(n=89)$ and Larimal $(n=71)$ both had a PCR-corrected cure rate of $100 \%$ at day 28 . Although parasite and fever clearance time was faster in the Larimal group, no significant difference was observed between the two drugs. No serious adverse effects were reported.

Conclusion: Five years after being introduced in Nigeria, both Coartem and Larimal have been shown to be safe and highly effective in the treatment of uncomplicated $P$. falciparum malaria in children.
\end{abstract}

Key words: ACTs; drug resistance; Nigeria; Plasmodium falciparum

J Infect Dev Ctries 2013; 7(12):975-982. doi:10.3855/jidc.3058

(Received 02 October 2012 - Accepted 04 February 2013)

Copyright $(0) 2013$ Ojurongbe et al. This is an open-access article distributed under the Creative Commons Attribution License, which permits unrestricted use, distribution, and reproduction in any medium, provided the original work is properly cited.

\section{Introduction}

The emergence and spread of drug-resistant Plasmodium falciparum continue to make malaria a major public health concern. Children and pregnant women living in tropical regions and non-immune travelers to malaria-endemic areas account for the highest morbidity and mortality resulting from malaria $[1,2]$. Due to the high level of parasite resistance to cheap and widely available chloroquine and sulfadoxine-pyrimethamine, artemisinin-based combination therapies (ACTs) have now replaced those failing drugs in order to combat the growing drug resistance. Artemisinin derivatives have short half-lives and are noted for their rapid reduction of parasite biomass resulting in a clinical and parasitological cure. An exception is at the ThaiCambodia border, where oral artemisinin-based monotherapies have been used for a long time [3,4], artemisinin resistance has not been reported elsewhere. Artemisinin is an effective replacement for chloroquine and sulfadoxine-pyrimethamine. The World Health Organisation (WHO) recommends that artemisinin should not be used as monotherapy but in combination with other drugs with long half-lives. The fixed-dose ACT, artemether-lumefantrine (Coartem) remains very effective and well tolerated. Other antimalarials such as amodiaquine, sulfadoxinepyrimethamine, and mefloquine are used as partner drugs for artemisinin to prevent the development of resistance. 
In 2005, the Nigerian government changed its policy for the treatment of uncomplicated malaria. Artemether-lumefantrine and artesunate-amodiaquine replaced chloroquine and sulfadoxine-pyrimethamine as the first line treatment [5]. Currently, artemetherlumefantrine (Coartem) and artesunate-amodiaquine (Larimal) are the most widely used among the ACTs currently available in Nigeria. A few years after the policy change, chloroquine and sulfadoxinepyrimethamine were still being widely used among the populace [6]. With increased efforts to raise awareness of appropriate malaria treatment, ACTs are now being widely used. Clinical trials of ACTs in different parts of Nigeria since its introduction have shown good efficacy and tolerability of ACTs in the treatment of uncomplicated malaria in children $[7,8]$. The high cure rates of ACTs have also been confirmed in different studies across sub-Saharan Africa [9].

Currently, ACT remains the most effective treatment for malaria; resistance to artemisinin and its derivatives would have a devastating effect on global malaria control. Constant diligent surveillance with the aim of monitoring the susceptibility of malaria parasites to artemisinin, its derivatives, and partner drugs in endemic areas is therefore essential. To date, no molecular marker has been identified for the monitoring of artemisinin and its derivatives [10]; therefore, effective monitoring still relies largely on in vivo studies with adequate follow-up.

Increased drug usage is known to significantly contribute to drug resistance. It is expected that as more people use a drug, the possibility that parasite will be exposed to inadequate drug levels will increase, thereby leading to selection of resistant parasites [11]. The aim of this study was to evaluate the effectiveness and safety of Coartem and Larimal in Nigerian children five years after their continuous usage.

\section{Methodology}

\section{Study site and patients}

This study was conducted in Ilesa, Osun state, located in the southwestern part of Nigeria. Malaria is present throughout the year with a marked increase during the raining season (i.e. April through September). Febrile children (between 6 and 144 months of age) attending the Wesley Guild Hospital, Ilesa (a unit of the Obafemi Awolowo University Teaching Hospitals Complex, Ile-Ife) were screened for $P$. falciparum parasitaemia. The initial screening for $P$. falciparum was conducted using the rapid diagnostic kit Paracheck (Orchid Biomedical Systems,
Goa, India). Children who tested positive for $P$. falciparum were recruited into the study. Other inclusion criteria included (1) no signs of severity or severe malaria (including severe anaemia defined by haemoglobin $<5 \mathrm{~g} / \mathrm{dL}$ ), (2) no intake of antimalarial drugs in the preceding four weeks, and (3) informed consent from the patients' parent or guardian. Thick blood films were prepared from a finger prick, stained with $10 \%$ Giemsa stain and examined using x 1000 magnification of a light microscope for confirmation and parasite quantification. In addition, approximately $10 \mu \mathrm{L}$ of blood was spotted on filter paper for parasite genotyping. Informed consent was obtained from each parent or legal guardian. Patients were excluded if they had signs of severe malaria, severe malnutrition, serious underlying diseases (renal, cardiac, or hepatic), or a known allergy to the study drugs. The study received ethical approval from the ethical review committee of the Obafemi Awolowo University Teaching Hospital Complex Ile Ife, Osun State, Nigeria.

\section{Study procedures}

This hospital-based open randomised clinical trial was conducted between February 2010 and September 2011, comparing three-day regimens of Coartem and Larima, with follow-ups after 28-days. Patients were enrolled, weighed, and randomised in blocks of 15 to receive Coartem or Larima. The number of patients included in this study was determined using the formula

$$
n=\frac{(a+b)^{2}\left(p_{1} q_{1}+p_{2} q_{2}\right)}{x^{2}}
$$

and a difference in the event rate of 0.10 was considered to be clinically relevant [12]. On the basis of a previous study in Nigeria, the cure rate of Coartem was estimated to be $96.5 \%$ [13]; the cure rate of artesunate-amodiaquine was estimated to be $97.9 \%$ [7]. The minimum sample size for each experimental arm was determined to be approximately 40 patients. All doses were administered under direct supervision of the investigators. In the event of vomiting within 30 minutes of taking the drug, the dose was repeated. If vomiting occurred between 30 and 60 minutes after administration, half the dose was given. Patients who kept vomiting were withdrawn and the drug was administered intravenously to them.

Drug therapy and follow-up

Coartem was administered as $20 \mathrm{mg}$ artemether/120 mg lumefantrine fixed-dose combination tablets (Novartis, Basel, Switzerland) 
twice daily as a three-day, six-dose regimen according to body weight (patients $5-<15 \mathrm{~kg}$ were given one tablet; patients $15-<25 \mathrm{~kg}$ were given two tablets); patients $25-<35 \mathrm{~kg}$ were given three tablets). The second dose was administered eight hours after the first dose, according to manufacturer's guideline. Larimal was administered as $50 \mathrm{mg}$ artesunate $153 \mathrm{mg}$ amodiaquine hydrochoride fixed dose combination tablets (Ipca, Mumbai, India) twice daily as a threedays, six-dose regimen according to body weight (patients $5-<15 \mathrm{~kg}$ were given one tablet); patients 15 $-<25 \mathrm{~kg}$ were given two tablets; patients $25-<35 \mathrm{~kg}$ were given three tablets). The second dose was administered eight hours after the first dose. Subsequent follow-up appointments were scheduled for days 3, 7, 14, 21 and 28; blood film was examined during the follow-up periods. Blood was also collected on filter paper during the follow-up periods. During the follow-up visits, parents or caregivers were asked about the child's well-being, behavioral and developmental concerns, as well as open questions about possible side effects that had occurred since the last visit. The study physicians examined the children during the follow-up visits. The primary effectiveness outcomes were the 28-days risks of recurrent symptomatic malaria unadjusted and adjusted by genotyping. The comparative tolerability of the drugs was assessed by the risk an adverse event (AE). A serious adverse event was defined as any AE that was life threatening, resulted in death or in a persistent or significant disability or incapacity, , or required hospitalization or significant medical intervention to prevent a serious outcome; this was assessed as recommended by WHO [14].

\section{Parasite genotyping}

Parasite genomic DNA was extracted from blood samples spotted on filter paper using QIAamp DNA blood kit (Qiagen, Hilden, Germany), according to the manufacturer's instructions. $P$ falciparum DNA were analyzed by nested polymerase chain reaction (PCR) using oligonucleotide primers for the conserved regions of merozoite surface protein 2 (MSP2) as well as oligonucleotides for FC27 and 3D7 specific family alleles, as described previously $[15,16]$.

\section{Data analysis}

Data were analyzed using JMP version 5 for Windows. The intention-to-treat group included all randomized participants who took at least one full dose, had one post-baseline efficacy assessment without any major protocol violations such as wrong dosage or wrong use of non-assigned drug, and completed follow-up until day 21. The per-protocol analysis included patients who received the three doses and had no major protocol violation up to day 28. Those who did not follow up or who withdrew consent were excluded from the per-protocol analysis. The primary endpoint was adequate clinical and parasitological response (ACPR), defined as the absence of parasitemia on day 28 irrespective of auxillary temperature without previously meeting the criteria for early treatment, late clinical failure, or late parasitological failure. The secondary endpoints were comparisons of the side effects and fever and parasite clearance of the two treatments. Groups were compared using the chi-square test or Fisher's exact test for qualitative outcome and the Student's t-test for quantitative outcome when applicable. A p-value (two-sided) of $<0.05$ was considered statistically significant.

\section{Results}

\section{Demographic data}

A total of 380 children were screened; 182 patients positive for $P$. falciparum infection determined by RDT and thick blood films that fulfilled inclusion criteria were randomized, as shown in Figure 1. There was no significant difference in the mean age of the children in the two study groups. There were no significant differences in the other baseline characteristics of the two treatment groups (Table 1).

Figure 1: Trial profile for the study

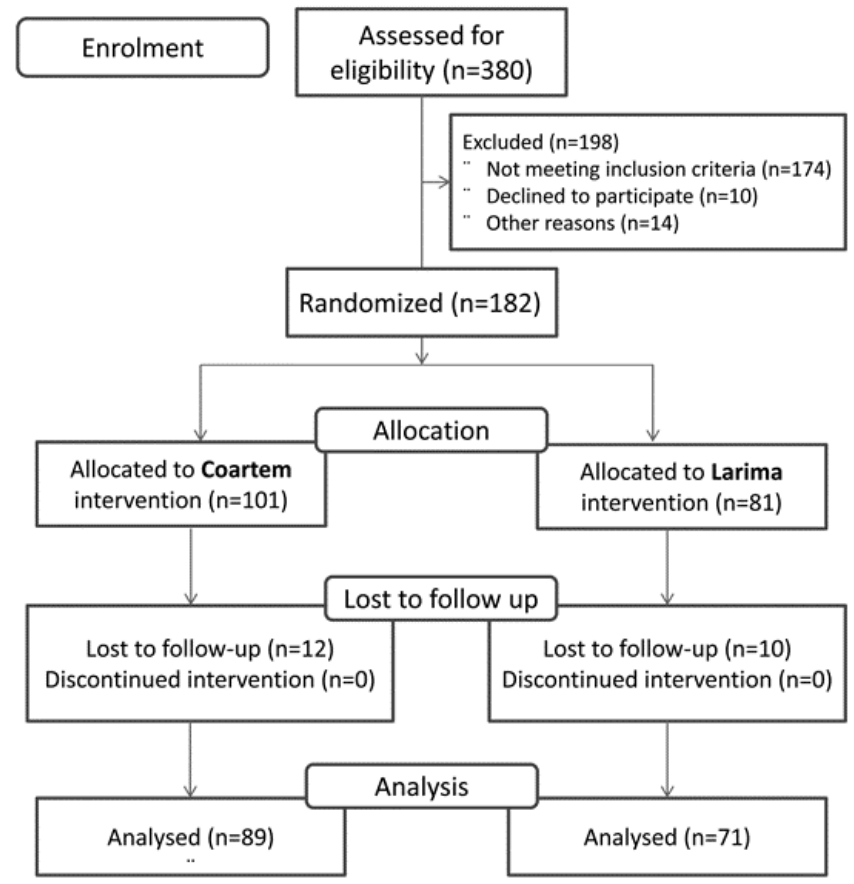


Table 1: Baseline characteristics of the study patients

\begin{tabular}{|c|c|c|c|}
\hline Characteristics & Coartem $(n=89)$ & Larimal $(n=71)$ & P-value \\
\hline Sex: Male : Female & $47: 42$ & $41: 29$ & 0.63 \\
\hline \multicolumn{4}{|l|}{ Weight $(\mathrm{Kg})$} \\
\hline Mean \pm SD & $14.4 \pm 7.4$ & $14.4 \pm 6.6$ & \multirow{3}{*}{0.99} \\
\hline Median & 12 & 12 & \\
\hline Range & $8-32$ & $8-39$ & \\
\hline \multicolumn{4}{|l|}{ Age (months) } \\
\hline Mean & $46.2 \pm 37.4$ & $45.6 \pm 37.2$ & 0.91 \\
\hline \multicolumn{4}{|l|}{ Temperature ${ }^{\circ} \mathrm{C}$} \\
\hline Mean \pm SD & $37.8 \pm 0.96$ & $38.0 \pm 0.94$ & \multirow{2}{*}{0.19} \\
\hline Median & 37.5 & 38.0 & \\
\hline \multicolumn{4}{|c|}{ Packed Cell Volume (\%) } \\
\hline Mean \pm SD & $32.2 \pm 5.9$ & $31.4 \pm 4.4$ & 0.31 \\
\hline \multicolumn{4}{|l|}{ Parasite density } \\
\hline Mean \pm SD & $4473.6 \pm 11019.2$ & $2402.3 \pm 2599.0$ & 0.07 \\
\hline Range & $40-56,480$ & $40-36,960$ & \\
\hline
\end{tabular}

Table 2: Treatment outcomes

\begin{tabular}{|c|c|c|c|}
\hline Outcome & Coartem & Larimal & P-value \\
\hline \multicolumn{4}{|l|}{ Intention to treat } \\
\hline Enrolled patients & 101 & 81 & \\
\hline Patient seen on day 28 & $89 / 101(88.1 \%)$ & $71 / 81(87.7 \%)$ & 1.00 \\
\hline Missing & $12 / 101(11.9 \%)$ & $10 / 81(12.3 \%)$ & 1.00 \\
\hline Crude parasitological failure D28 & $2 / 101(2.0 \%)$ & $0 / 81(0 \%)$ & 0.54 \\
\hline PCR-adjusted failure D28 & $0 / 101(0 \%)$ & $0 / 81(0 \%)$ & - \\
\hline PCR-adjusted cure rate D28 & 99/101 (98.0\%) & $81 / 81(100 \%)$ & 0.54 \\
\hline \multicolumn{4}{|l|}{ Per-protocol analysis } \\
\hline Patient seen on day 28 & 89 & 71 & \\
\hline Crude parasitological failure D28 & $2 / 89(2.3 \%)$ & $0 / 71(0 \%)$ & 0.54 \\
\hline PCR-adjusted failure D28 & $0 / 89(0 \%)$ & $0 / 71(0 \%)$ & - \\
\hline PCR-adjusted cure rate D28 & $89 / 89(100 \%)$ & $71 / 71(100 \%)$ & - \\
\hline
\end{tabular}

Table 3: Mild and adverse reactions reported from D0 to D28

\begin{tabular}{lcc}
\hline Symptoms & Coartem $(\mathbf{n}=\mathbf{1 0 1})$ & Larimal $(\mathbf{n}=\mathbf{7 1})$ \\
\hline Vomiting & $20(19.8 \%)$ & $11(20 \%)$ \\
Diarrhoea & $5(5.0 \%)$ & $4(7.3 \%)$ \\
Abdominal pain & $4(4.0 \%)$ & $5(9.1 \%)$ \\
Allergy (scratching, catarrh) & $15(14.8 \%)$ & $12(21.8 \%)$ \\
Cough & $23(22.8 \%)$ & $12(21.8 \%)$ \\
Headache & $9(8.9 \%)$ & $4(7.2 \%)$ \\
\hline
\end{tabular}


Figure 2: Parasite clearance time in the two study groups. The figure shows the percentage reduction of parasitaemia from the recruitment day (day 0) over the follow-up period of 28 days according to the per-protocol analysis

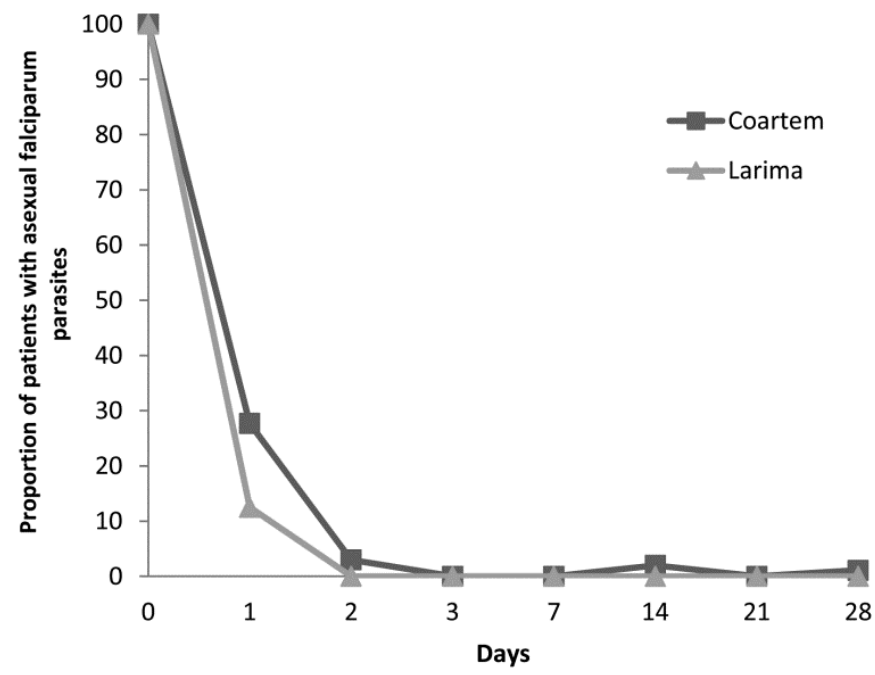

\section{Therapeutic efficacy}

Adjusted and unadjusted treatment outcome was available for 182 children in the intention-to-treat (ITT) and for 160 children in the per-protocol (PP) analysis. No early treatment failure (ETF) was observed in either study group. Adequate clinical and parasitological responses were similarly high in both treatment groups (Table 2). Two children in the Coartem study group had crude parasitological failure on day 28 and were classified as new infections after confirmation with PCR; no crude parasitological failure was observed in the Larimal treatment group. In the ITT and PP analysis, the PCR-adjusted cure rate on day 28 was $100 \%$ for both drugs.

\section{Parasite and fever clearance}

The number of days to clear parasites was similar in the two groups, but relatively faster in the Larimal group (Figure 2). No significant difference was observed in fever clearance time between the two groups, although the number of fever cases was lower in the Larimal group after 24 hours. After 48 hours, there were no fever cases in either group (data not shown).

\section{Tolerability and safety}

Overall, Coartem and Larimal were well tolerated in this study. None of the patients presented with serious adverse events that necessitated withdrawal from the study or hospitalization. Adverse effects associated with the gastrointestinal tract such as vomiting (20\%), diarrhoea $(5.8 \%$,) and abdominal pain
$(5.9 \%)$ were the most common effects considered to be treatment-related by the investigators. Allergies (scratching) also appeared in $17.3 \%$ of the patients. Overall, adverse events were more frequent in the Larimal group $(21.8 \%)$ compared to the Coartem group $(14.8 \%)$, but the difference was not significant (Table 3).

\section{Discussion}

The present study evaluated the efficacy of Coartem and Larimal in Nigeria five years after their introduction as first-line antimalarial treatments. Overall, the study showed a high efficacy of the two forms of ACTs in Nigerian children; the PCR-adjusted cure rate on day 28 was $100 \%$ in both treatment groups. These results are in agreement with earlier studies in Nigeria that have shown both combinations to be safe and well tolerated $[7,8,17]$ and are also consistent with the high cure rates that have been reported for ACT in other malaria-endemic areas of Africa [18-20]. Artemisinin and related endo-peroxide antimalarials act rapidly by clearing $P$. falciparum in the blood, thereby providing prompt therapy for severe infections [21]. Moreover, artemisinins are also active against parasites resistant to two major classes of antimalarials, quinolines and antifolates, thereby making them the most potent and effective antimalarials presently used [22]. As a drug that has a very short half-life, artemisinin cannot be used as monotherapy to treat uncomplicated malaria due to frequent recrudescence of infections after standard three-day treatments [23]. Therefore, the combination of artemisinin with longer-acting drugs is recommended to effectively treat uncomplicated malaria. Currently, artemether-lumefantrine and artesunate plus amodiaquine are the most widely available artemisinin combination drugs produced on a large scale with good manufacturing practices (GMP) [20], and they have both been shown to have comparative efficacy in the treatment of all forms of malaria in sub-Saharan Africa. However, there have been some contrasting reports on the comparative performance of these drugs. Some studies have reported a marginally higher day 28 PCR-corrected cure rate for artemether-lumefantrine over artesunate plus amodiaquine as opposed to others. Generally, studies from eastern Africa $[18,24]$ seem to suggest that artemether-lumefantrine has more clinical benefits than artesunate plus amodiaquine, while studies from western Africa [17,20] show that artesunate plus amodiaquine tend to have marginally better efficacy rates over artemether-lumefantrine. The reason for this 
observation needs to be further investigated. In the current study, cure rates on day 28 in ITT and PP analysis demonstrate the efficacy of both Coartem and Larimal in the treatment of uncomplicated malaria in Nigerian children. However, two re-infection cases were reported on the Coartem arm on day 28.

In the current study, the clinical safety in terms of adverse events and clinical laboratory findings were comparable in Coartem and Larimal, with no evidence of haematological and hepatic toxicity associated with the use of amodiaquine [25]. No serious adverse effects were observed in either treatment group, and the drugs were well tolerated, with comparable profiles. All the adverse effects reported resolved without needing any special treatment except for the use of analgesic (Paracetamol) for pain, antihistamine for allergies, and oral rehydration therapy (ORT) for diarrhoea. Larimal was well tolerated; only minor changes in safety parameters occurred, although the sample size may not have been sufficient to detect rare events. There was no indication of liver or hematological toxicity, the two toxicities that have caused fatalities in the past when amodiaquine was used as a prophylaxis [26,27]. Fever and parasite clearance was comparable between the two treatment groups with both resolving rapidly within 48 hours of the initiation of treatment.

A high prevalence of the $P$. falciparum chloroquine-resistant transporter (pfcrt T76) gene and the $P$. falciparum multidrug (pfmdr1 Y86) gene has previously been reported in the study area $[28,29]$, which could indicate the presence of potentially amodiaquine-resistant parasites [30]. This may raise a question about the long-term utility of amodiaquine as a partner drug for ACT in Nigeria. Although the impact of mutant pfert T76 and pfmdr1 Y86 allele prevalence on the treatment outcome in the study could not be assessed because there was no treatment failure, the artesunate plus amodiaquine combination has been associated with a high cure rate in areas where pfcrt K76T alleles are prevalent [31,32]. Thus, the argument remains about whether the pfcrt and pfmdr1 chloroquine-resistant molecular markers can also be used as resistance markers for amodiaquine. A recent study in Burkina Faso [33] observed a weak association of $\mathrm{pfcrt} / \mathrm{pfmdr} 1$ alleles with amodiaquine resistance, suggesting that these resistance markers may not be considered as an alternative to clinical trials in the evaluation of amodiaquine plus artesunate in the area. A suitable drug resistance marker has not yet been identified for the ACTs, and the monitoring of the efficacy of the drug will still have to rely largely on in vivo efficacy studies.

\section{Conclusion}

Five years after the introduction of ACTs in Nigeria, the two most commonly use ACTs still remain effective and safe for the treatment of uncomplicated falciparum malaria in children. While the search for effective malaria vaccine and vector control measures that will completely eradicate malaria in the sub-Saharan Africa continues, there is need for up-to-date, dynamic, and comparative data on anti-malarial drug efficacy and safety as chemotherapy still remains the cornerstone of malaria control.

\section{Acknowledgements}

We would like to thank all the children and parents and guardians for their cooperation. We also thank the nurses at the Department of Paediatrics and Child Health OAUTH Ilesa for their assistance. Vital efforts of the laboratory team (Adeola Ayileka and Peter Awosanmi) are warmly acknowledged. We also acknowledge the input of Dr. Benjamin Mordmueller of ITM Tuebingen, Germany. Olusola Ojurongbe was supported by Alexander von Humboldt foundation Germany.

\section{References}

1. Kitua A, Ogundahunsi O, Lines J, Mgone C (2011) Conquering malaria: enhancing the impact of effective interventions towards elimination in the diverse and changing epidemiology. J Glob Infect Dis 3: 161-165.

2. Wilson ME, Weld LH, Boggild A, Keystone JS, Kain KC, von Sonnenburg F, Schwartz E, GeoSentinel Surveillance Network (2007) Fever in returned travelers: results from the GeoSentinel Surveillance Network. Clin Infect Dis Off Publ Infect Dis Soc Am 44: 1560-1568.

3. Alker AP, Lim P, Sem R, Shah NK, Yi P, Bouth DM, Tsuyuoka R, Maguire JD, Fandeur T, Ariey F, Wongsrichanalai C, Meshnick SR (2007) Pfmdr1 and in vivo resistance to artesunate-mefloquine in falciparum malaria on the Cambodian-Thai border. Am J Trop Med Hyg 76: 641647.

4. Dondorp AM, Nosten F, Yi P, Das D, Phyo AP, Tarning J, Lwin KM, Ariey F, Hanpithakpong W, Lee SJ, Ringwald P, Silamut K, Imwong M, Chotivanich K, Lim P, Herdman T, An SS, Yeung S, Singhasivanon P, Day NP, Lindegardh N, Socheat D, White NJ (2009) Artemisinin resistance in Plasmodium falciparum malaria. N Engl J Med 361: 455-467

5. Federal Ministry of Health (2005) National antimalarial treatment policy.

6. Ogungbamigbe TO, Ojurongbe O, Ogunro PS, Okanlawon BM, Kolawole SO (2008) Chloroquine resistant Plasmodium falciparum malaria in Osogbo Nigeria: efficacy of amodiaquine + sulfadoxine-pyrimethamine and chloroquine + chlorpheniramine for treatment. Memórias Inst Oswaldo Cruz 103: 79-84. 
7. Ayede IA, Falade AG, Sowunmi A, Jansen FH (2010) An open randomized clinical trial in comparing two artesunatebased combination treatments on Plasmodium falciparum malaria in Nigerian children: artesunate/sulphamethoxypyrazine/pyrimethamine (fixed dose over 24 hours) versus artesunate/amodiaquine (fixed dose over 48 hours). Malar J 9: 378.

8. Falade CO, Ogunkunle OO, Dada-Adegbola HO, Falade AG, de Palacios PI, Hunt P, Virtanen M, Oduola AM, Salako LA (2008) Evaluation of the efficacy and safety of artemetherlumefantrine in the treatment of acute uncomplicated Plasmodium falciparum malaria in Nigerian infants and children. Malar J 7: 246.

9. Thwing J, Eisele TP, Steketee RW (2011) Protective efficacy of malaria case management and intermittent preventive treatment for preventing malaria mortality in children: a systematic review for the Lives Saved Tool. BMC Public Health 11 Suppl 3: S14.

10. Imwong M, Dondorp AM, Nosten F, et al (2010) Exploring the contribution of candidate genes to artemisinin resistance in Plasmodium falciparum. Antimicrob Agents Chemother 54: 2886-2892.

11. Watkins WM, Mosobo M (1993) Treatment of Plasmodium falciparum malaria with pyrimethamine-sulfadoxine: selective pressure for resistance is a function of long elimination halflife. Trans R Soc Trop Med Hyg 87: 75-78.

12. Noordzij M, Tripepi G, Dekker FW, Zoccali C, Tanck MW, Jager KJ (2010) Sample size calculations: basic principles and common pitfalls. Nephrol Dial Transplant Off Publ Eur Dial Transpl Assoc - Eur Ren Assoc 25: 1388-1393.

13. Gbotosho GO, Sowunmi A, Happi CT, Okuboyejo TM (2011) Therapeutic efficacies of artemisinin-based combination therapies in Nigerian children with uncomplicated falciparum malaria during five years of adoption as first-line treatments. Am J Trop Med Hyg 84: 936-943.

14. Edwards IR, Biriell C (1994) Harmonisation in pharmacovigilance. Drug Saf Int J Med Toxicol Drug Exp 10: 93-102.

15. Ojurongbe O, Fagbenro-Beyioku AF, Adeyeba OA, Kun JF (2011) Allelic diversity of merozoite surface protein 2 gene of $P$ falciparum among children in Osogbo, Nigeria. West Indian Med J 60: 19-23.

16. Ntoumi F, Contamin H, Rogier C, Bonnefoy S, Trape JF, Mercereau-Puijalon O (1995) Age-dependent carriage of multiple Plasmodium falciparum merozoite surface antigen-2 alleles in asymptomatic malaria infections. Am J Trop Med Hyg 52: 81-88.

17. Michael OS, Gbotosho GO, Folarin OA, Okuboyejo T, Sowunmi A, Oduola AMJ, Happi CT (2010) Early variations in plasmodium falciparum dynamics in Nigerian children after treatment with two artemisinin-based combinations: implications on delayed parasite clearance. Malar J 9: 335.

18. Kabanywanyi AM, Mwita A, Sumari D, Mandike R, Mugittu K, Abdulla S (2007) Efficacy and safety of artemisinin-based antimalarial in the treatment of uncomplicated malaria in children in southern Tanzania. Malar J 6: 146.

19. Faye B, Offianan AT, Ndiaye JL, Tine RC, Touré W, Djoman K, Sylla K, Ndiaye PS, Penali L, Gaye O (2010) Efficacy and tolerability of artesunate-amodiaquine (Camoquin plus) versus artemether-lumefantrine (Coartem) against uncomplicated Plasmodium falciparum malaria: multisite trial in Senegal and Ivory Coast. Trop Med Int Heal TM IH 15: 608-613.
20. Kobbe R, Klein P, Adjei S, Amemasor S, Thompson WN, Heidemann H, Nielsen MV, Vohwinkel J, Hogan B, Kreuels B, Bührlen M, Loag W, Ansong D, May J (2008) A randomized trial on effectiveness of artemether-lumefantrine versus artesunate plus amodiaquine for unsupervised treatment of uncomplicated Plasmodium falciparum malaria in Ghanaian children. Malar J 7: 261.

21. Enserink M (2010) Malaria's drug miracle in danger. Science 328: 844-846.

22. Klonis N, Crespo-Ortiz MP, Bottova I, Abu-Bakar N, Kenny S, Rosenthal PJ, Tilley L (2011) Artemisinin activity against Plasmodium falciparum requires hemoglobin uptake and digestion. Proc Natl Acad Sci U S A 108: 11405-11410.

23. Eastman RT, Fidock DA (2009) Artemisinin-based combination therapies: a vital tool in efforts to eliminate malaria. Nat Rev Microbiol 7: 864-874.

24. Mårtensson A, Strömberg J, Sisowath C, Msellem MI, Gil JP, Montgomery SM, Olliaro P, Ali AS, Björkman A (2005) Efficacy of artesunate plus amodiaquine versus that of artemether-lumefantrine for the treatment of uncomplicated childhood Plasmodium falciparum malaria in Zanzibar, Tanzania. Clin Infect Dis Off Publ Infect Dis Soc Am 41: 1079-1086.

25. Ndiaye J-LA, Faye B, Gueye A, Tine R, Ndiaye D, Tchania C, Ndiaye I, Barry A, Cissé B, Lameyre V, Gaye O (2011) Repeated treatment of recurrent uncomplicated Plasmodium falciparum malaria in Senegal with fixed-dose artesunate plus amodiaquine versus fixed-dose artemether plus lumefantrine: a randomized, open-label trial. Malar J 10: 237.

26. Taylor WRJ, White NJ (2004) Antimalarial drug toxicity: a review. Drug Saf Int J Med Toxicol Drug Exp 27: 25-61.

27. Navaratnam V, Ramanathan S, Wahab MSA, Siew Hua G, Mansor SM, Kiechel J-R, Vaillant M, Taylor WRJ, Olliaro P (2009) Tolerability and pharmacokinetics of non-fixed and fixed combinations of artesunate and amodiaquine in Malaysian healthy normal volunteers. Eur J Clin Pharmacol 65: 809-821.

28. Ojurongbe O, Oyedeji SI, Oyibo WA, Fagbenro-Beyioku AF, Kun JF (2010) Molecular surveillance of drug-resistant Plasmodium falciparum in two distinct geographical areas of Nigeria. Wien Klin Wochenschr 122: 681-685.

29. Ojurongbe O, Ogungbamigbe TO, Fagbenro-Beyioku AF, Fendel R, Kremsner PG, Kun JF (2007) Rapid detection of Pfcrt and Pfmdr1 mutations in Plasmodium falciparum isolates by FRET and in vivo response to chloroquine among children from Osogbo, Nigeria. Malar J 6: 41.

30. Folarin OA, Bustamante C, Gbotosho GO, Sowunmi A, Zalis MG, Oduola AMJ, Happi CT (2011) In vitro amodiaquine resistance and its association with mutations in pfert and pfmdrl genes of Plasmodium falciparum isolates from Nigeria. Acta Trop 120: 224-230.

31. Gbotosho GO, Sowunmi A, Happi CT, Okuboyejo TM (2011) Therapeutic efficacies of artemisinin-based combination therapies in Nigerian children with uncomplicated falciparum malaria during five years of adoption as first-line treatments. Am J Trop Med Hyg 84: 936-943.

32. Zongo I, Dorsey G, Rouamba N, Tinto H, Dokomajilar C, Guiguemde RT, Rosenthal PJ, Ouedraogo JB (2007) Artemether-lumefantrine versus amodiaquine plus sulfadoxine-pyrimethamine for uncomplicated falciparum malaria in Burkina Faso: a randomised non-inferiority trial. Lancet 369: 491-498. 
33. Danquah I, Coulibaly B, Meissner P, Petruschke I, Müller O, Mockenhaupt FP (2010) Selection of pfmdr1 and pfcrt alleles in amodiaquine treatment failure in north-western Burkina Faso. Acta Trop 114: 63-66.

\section{Corresponding author}

Ojurongbe Olusola

Department of Medical Microbiology \& Parasitology

Ladoke Akintola University of Technology

PMB 4400, Osogbo

Osun State, Nigeria

Email: oojurongbe@lautech.edu.ng

Conflict of interests: No conflict of interests is declared. 